\title{
Artificial intelligence, its impact on innovation, and the Google effect
}

\author{
Subhas Sikdar ${ }^{1}$
}

Published online: 20 December 2017

c) Springer-Verlag GmbH Germany, part of Springer Nature 2017

Users of devices such as Apple Computer's Siri or Amazon's Alexa are familiar with the way intelligent machines communicate with humans with voice- and thought recognition. The clarity of diction, and thought compositions of these machines remind us of the exploits of the supercomputer Hal 9000 of Arthur C. Clark's 2001: a space odyssey, as depicted in Stanley Kubrick's movie of the same name. Hal's voice quality was certainly not as human-like as Alexa's, for instance, but Hal displayed distinct emotive qualities not yet seen in these familiar commercial devices. Information technology's data processing power has reached a point that self-driving vehicles appear imminent, as all major automotive companies have announced making autonomous cars and trucks to be available in the near future. Google has extensively road-tested passenger vehicles for several years, and Tesla is about to start selling such cars. Even autonomous taxis have been announced. All these developments can be explained as instant decision making, resulting from algorithms that are able to process massive amounts of provided and updated data in nanoseconds. The Silicon Valley futurist and innovator Ray Kurzweil ${ }^{1}$ years ago predicted that thinking machines will match and then surpass human thinking. Singularity was the term used for this point of no return. Kurzweil arrived at this conviction by merging his own research on artificial intelligence with advances in neuroscience. ${ }^{2}$ These developments are supremely beneficial to us in many aspects of our living standards. This may not remain so according to some prognosticators.

Prominent innovators from the Silicon Valley have recently been sounding alarm that artificial intelligence has progressed to the point that intelligent machines on their own can evolve into thinking machines to pose grave danger to humans. They can be termed Franken-machines. The great physicist Stephen Hawking has also sounded similar

Subhas Sikdar-Editor in Chief.

Subhas Sikdar

subhas.sikdar@gmail.com

1 Cincinnati, OH, USA alarms. In simple terms, a role reversal is imminent when we become subservient to machines rather than the reverse. In a tussle between human mind and a present-day Hal, for example, Hal will come out as victorious. This is ominous even if the effect is entirely beneficial, which obviously would not be. But will it come to pass? Is that the end of story? Recently, it has also been argued that we would need and will have the technology to enhance our brainpower to tackle the accelerating evolution of artificial intelligence. This would be achieved by planting inserts into the human brain to augment its processing power. ${ }^{3}$

We can argue that artificial intelligence of digital technology thus far is superfast in information processing and all the exploits of the extant machines merely are a response to information fed as instruction. In this sense, our interaction with Alexa is not really a conversation, as Alexa cannot tell us anything about what she has not been provided as data or has not surreptitiously gathered by listening to us. On the other hand, when you order an article of commerce through a vendor's website, frequently you receive suggestions with this kind of message: buyers who ordered this are also interested in the following. A clever marketing trick alright, but behind it is an algorithm that looks at your profile of preference and makes an informed suggestion. Some of the choices given to you may be on target, but others are off the mark. But in situations where the choice matters a great deal, we cannot take a chance by choosing one of the offerings. Suppose we want to apply the art of artificial intelligence to the design of environmentally preferable processes or products. Such efforts are already under way in the form of algorithms for process integration, which is able to satisfy certain objectives of, say energy use minimization, in a process flowsheet. Process integration algorithms can satisfy this objective in the face of random or unexpected changes in the values of the process variables. This achievement,

\footnotetext{
${ }^{1}$ Krzweil, Ray, Singularity is near.

${ }^{2}$ Kurzeil, Ray, How to create a mind, Viking Penguin, New York, New York, 2012.

${ }^{3}$ Carr, Nicholas, Wall Street Journal, Review section, October 7, 2017.
} 
while welcome, is similar to a self-driving car that needs to navigate the vagaries of street traffic and signals. But can we train a suitable intelligent computer in engineering principles so that it can replace a human engineer to uniquely design a process or a product to specifications, or even to determine the specifications?

Also, we need to think of the consequence of using these awesome machines. The scariest consequence of artificial intelligence has been sounded by Elon Musk of Tesla Motors and supported by Stephen Hawking. Since technologies have social consequences, what would happen if we are able to achieve driverless public transportation, robots serving in the supermarkets and restaurants and similar developments throughout social activities? In the past we saw the effects of vanishing horse-drawn carriages, and computers doing our accounting and finances, etc. Instead of massive unemployment widely predicted, many more new jobs were created than jobs that disappeared. In the event of accelerating innovations that replace human labor, will we see more needs of human labor, not less? Will past be the prologue?

Another social effect is already looking ominous. While digital technology has created devices for exponential and instant communications using the smart phones, we communicate through the machines, in preference to talking to each other. Instead of bringing us together, information technology is dividing us into groups of similarly thinking individuals. Anyone familiar with Facebook would be able to attest to this truth. This has been termed the Google effect. Technologically oriented amongst us would refrain from sounding like the luddites at the beginning of the industrial revolution, but it is difficult to dismiss the warning signs. As Matt Ridley mentions in his latest book, ${ }^{4}$ Francis Crick of the DNA structure fame, thought that once the full implication of the function of genetic material is explored and understood, we will observe that mind is essentially the result of material evolution, in sync with the belief of the philosopher Spinoza. Not mind over matter; mind is matter. One thing is for sure: we are about to see the real-world ramifications of some of the coming attractions. Will we lose control on our fate? Or, just a few very technology-savvy individuals will control the rest of us? The answers to the questions raised here will slowly but surely be revealed in coming years.

${ }^{4}$ Ridley, Matt, The Evolution of Everything, HarperCollins, New York, 2015. 